\title{
PRESERVATION AND PROGRESS: \\ USING TIBETAN MEDICINE AS A MODEL TO DEFINE \\ A PROGRESSIVE ROLE FOR TRADITIONAL ASIAN \\ MEDICINE IN MODERN HEALTHCARE
}

\author{
ELIOT TOKAR
}

\begin{abstract}
The history of Tibetan medicine's development provides an excellent model for planning the appropriate utilisation of 'foreign' medical systems and traditions within a new culture that incorporates progressive approaches for the use of both medical pluralism and integration. This history takes on increasing relevance in the modern world as the practice of Tibetan medicine and other forms of traditional Asian medicine have been spreading in the West where they have been faced with commoditisation and the hegemony of biomedicine. In the face of modern globalization, certain key questions have yet to be sufficiently addressed. How can systems of traditional Asian medicine be incorporated into western cultures in a manner that permits them to contribute to our understanding of health and disease while allowing them to retain their own integrity? How can they be utilised to aid in the solution of public health problems that exist in the West? What should be the proper interaction between traditional Asian medical systems (e.g., Tibetan medicine) and biomedicine? To determine the answer to these and other vital questions, we must take into account the many cultural, political, economic and scientific issues that affect the state of both public health and individual healthcare.
\end{abstract}

The history of Tibetan medicine provides us with an important progressive model for thinking about how Asian medical systems and traditions can maintain their integrity while continuing to develop in the context of modern global culture. This example takes on special relevance as the practices of Asian medical systems and traditions have been spreading in the West, and especially since the EU, the US and the WHO have begun to exert hegemony over how they are to be regarded internationally. ${ }^{1}$

1 Allen 2006; Miller 2004.

(C) Brill, Leiden, 2006

ASME 2,2 
Tibetan medicine's development provides a precedent for understanding how medical pluralism can be a vital tool for achieving progress in global healthcare. If ancient medical traditions are allowed to preserve their disciplines intact, while continuing to develop to meet evolving public health circumstances, they can provide in the aggregate much of what is needed to solve a broad range of existing health problems. Simply put, if modern and traditional medical approaches can coexist, achieve parity and, when appropriate, learn to collaborate, our understanding of health and the treatment of disease will become greater. The resultant wisdom will give lay people and medical professionals greater freedom of choice as to how to prevent and remedy illness in a manner that is more humanistic, ecologically centred and economically feasible. It will also provide the basis for approaches to medical integration that are based upon sound principles, rather than upon the economic priorities of modern industrialised medicine.

For several decades, traditional Asian medical disciplines have been increasingly practised in the West. In the US some, such as Chinese medicine, have gained wide acceptance, while others, such as Tibetan and Ayurvedic medicine, are still emerging. However, certain key questions regarding this medical and cultural phenomenon have generally not been given the attention that they deserve. At medical and scholarly conferences, there are regular discussions of the historical, anthropological, botanical, medical and epistemological aspects of these disciplines. It is urgent, however, that we bring to the forefront of current discussions the question of how we can use the new international interest in traditional Asian medical practices to help ensure their survival and progress along traditional lines, even while they serve to contribute to our understanding of health and disease and benefit those suffering with illness.

Some of the questions that we need to address include: How should these systems and traditions of medicine best be incorporated into global culture? How can Asian medical systems and traditions be utilised to aid in the solution of significant public health problems, such as the currently acknowledged American healthcare crisis? What progressive alternatives are there to existent biomedicallyoriented complementary or integrative models, for the interaction between systems of traditional Asian medicine and biomedicine?

A Tibetan medicine doctor's central concern is the individual wellbeing of his or her patients. In Tibetan medical theory, however, 
many spiritual, cultural, political, and economic issues are recognised and/or implied as having a direct affect upon the health of individuals and, therefore, public health overall. In a recent letter to the Editor published in the New York Times, I wrote that 'the redefining of medicine in America as a healthcare industry, where economics and consumerism are the central issue rather than health, undermines our attempts to find real solutions to the continual growth of degenerative illness'. I further asserted that, if medicine can reclaim its traditional role in society, and greater medical pluralism is realised, then we will be better able to begin to change our focus away from the commerce of disease and towards an understanding of how to achieve health'. ${ }^{2}$

Many people, worldwide, are unnecessarily handicapped in achieving such understanding because biomedicine provides a very limited definition of health. By defining health largely as the absence of disease, biomedicine, and the cultural view that it perpetuates, leaves us stranded with a simplistic view of a complex and primary component of our existence. ${ }^{3}$ Attempts to define health more broadly fall short because 'a complete state of physical, mental and social well-being', such as that described by the World Health Organisation, is not clearly quantifiable in biomedical terms. ${ }^{4}$ In order for any of the vast and varied fields of traditional Asian medicine to make a significant contribution to solving healthcare crises, we must first be able to inculcate clear, detailed and practical definitions of 'health' into global culture.

\section{Tibetan medical wisdom}

The process of introducing new ideas regarding health and medicine, and creating a genuinely integrative paradigm, is clearly present in the history of Tibetan medicine. In the fourth century, Tibetans began encouraging the introduction of new ideas regarding medicine into their country. Over the centuries that followed, they gained influences from India, Persia, China and Greece, freely allowing medical knowledge from these cultures to mix with their own indigenous medicine.

\footnotetext{
2 Tokar 2005, p. A22.

${ }^{3}$ Glanze et al. 1992 p. 306; http://www2.merriam-webster.com/cgi-bin/mwmednlm? book=Medical\&va=health

+ WHO 2002.
} 
In the eighth century, the Tibetan government sponsored conferences at which doctors skilled in foreign medical traditions presented and debated their ideas regarding health and the treatment of illness. Those with superior knowledge and abilities were invited to stay and thereby promote medical pluralism in Tibet. Through a process that took centuries, this knowledge was slowly integrated creating a unique medical discipline. In the thirteenth century, all of the seminal Tibetan medical texts were collected, and the current version of the central text of Tibetan medicine, the rGyud zhi, was composed. ${ }^{5}$

The medical system presented in these texts was singular, yet also a synthesis of the major medical systems of the ancient world. These medical traditions developed an ecological and qualitative approach to researching and classifying the phenomena that comprise the natural world. This type of scientific method provides us with a set of information about our world that is wholly different from that which is observed by modern science's own profound materialist, quantitative and reductionist approaches.

Ancient scientific theories explain how the forces of nature are directly correlated with, and thereby influence, the functioning of the human organism. The 'physics' of Tibetan medicine, for example, utilises a qualitatively based system of analysis and categorisation in order to define those basic forces of nature in the Tibetan theory of the five elements. The characteristics, and therefore the nature of all matter and energy, then result from the specifically delineated qualities of these elements as they manifest individually or in combination.

A central aspect of Tibetan medical theory are the nyes pa (pron. nyae pa), the three principles of function of the body and mind; [rlung (pron. loong), mkhris pa (pron. tree pa), and bad kan (pron. pay gen).] The nyes $p a$ are of course somewhat similar to the three doshas in Ayurvedic medicine. These three nyes $p a$ are expressions of the elements that occur within our organism and determine the proper functioning of our body and mind. Each of the three principles has a distinct role in physical and mental function, as well as a specific set of relationships to our body's organs, constituents (e.g., blood, tissue, etc.) and to the processes of our physiological systems (e.g., the nervous and circulatory systems) and to our mind.

Theories regarding such principles are common to many systems of traditional Asian medicine (e.g., qi, jing, and shen in Chinese medicine

${ }^{5}$ Trogawa 1984-9; Yonten 1989 pp. 32-51; rGyud zhi 1994. 
or the dosha in Ayurvedic medicine). Traditional natural medicine places great emphasis upon understanding the role of these principles in the creation and maintenance of the functions of the body's organs, systems and substances. The recognition of the existence of these principles, and their central importance as the underlying basis for all of the functions of our body and mind, clearly distinguishes the perspective of Asian medical systems and traditions from that of biomedicine.

Understanding the function of the three nyes $p a$, for example, also allows us to comprehend the physical principles that are the basis of the interdependence that exists between our body and mind that is depicted in Tibetan medicine. Tibetan medical theory explains that our nyes $p a$ are created as a direct result of an interaction between our mind's developmental process and the five physical elements at various stages of development in the womb. Tibetan medicine's detailed description of that genesis provides a model that explains how consciousness begins to play a direct role in physical function from the very early stages of embryologic development. ${ }^{6}$

The recognition of such principles and their primary role in physiology allows for the detailed definition of health that is described by traditional Asian medical systems, and which is largely missing in biomedicine. A disequilibrium occurring in the natural ecology of the nyes $p a$ is understood to lead to dysfunction and, if not treated, to illness and disease. Herein lies the basis upon which the discovery of the causes of illness becomes the central consideration in the diagnosis and treatment of disease in Tibetan medicine. In studying Tibetan medicine, one first learns the nature of the properly functioning human organism - that is, the definition of health-prior to studying about the cause and treatment of disease. In Tibetan medicine, it would be illogical to attempt to understand illness without first clearly understanding the natural, or healthy, state of the body and mind.?

From this theory of the three principles of function, Tibetan medicine is able to deduce the ways in which health is directly affected by all aspects of behaviour in its various physical, psychological and spiritual forms. The direct relationship of the five elements to the three nyes pa also gives rise to Tibetan medicine's ability to evaluate

${ }^{6}$ Parfionovitch 1992, pp. 25-6, 181-2; Dhonden 2000 pp. 49-50.

7 Dhonden 2000 pp. 21-63. 
the specific effects of diet on health. It provides a means with which to analyse the qualities of foods relative to their elemental characteristics, such as their tastes, and to correlate them with the functioning of the three nyes pa. The same is true regarding Tibetan medicine's ability to comprehend and evaluate the medical significance of regional and seasonal climates. ${ }^{8}$

Given the breadth of the health-related interdependencies that it comprehends, public health could benefit greatly if Western people understood the detailed view of both health and the aetiology of disease that is depicted in Tibetan medicine's theory regarding the role of the nyes pa. Preventive medicine would gain a deeper meaning if there were a greater appreciation of how imbalances, occurring in the homeostasis of the three principles of function, lead to dysfunction within the body's physical and mental processes. When these physiological processes break down our body's organs, systems and biochemistry are directly impacted. When such imbalances begin to occur, they are unfortunately often not readily diagnosable by biomedicine, whether or not they are immediately manifested symptomatically. If left unchecked, however, they are the cause of the development of illness and disease.

If the public was educated regarding these principles, or for that matter, concerning similar concepts existing in all other forms of traditional Asian medicine, they could begin to prevent many diseases at their earliest stages of development. It would therefore benefit the progress of public health if education regarding preventive medicine and natural health began at the elementary school level. In this way, our modern society could begin to replicate the benefits of that natural medical knowledge that was traditionally perpetuated through folk culture.

In the midst of our overly specialised modern culture, we should understand that the first line of medical care among traditional people was and still is often not a physician but, for example, one's mother or grandmother. The physician had a distinct role, but much important primary medical care and health knowledge was delivered through the family and community. Re-empowering lay people regarding their health through natural and preventive medical education was, in fact, one of the central goals of the American grassroots

${ }^{8}$ Donden 1986, pp. 131-86. 
alternative medicine movement that perpetuated itself for roughly three decades beginning in the 1960s.

By disseminating information about traditional Asian medicine's detailed ecological and qualitative view of health, we would be offering people tools with which they could create individualised approaches to their behaviour, diet and environment, and that could help them prevent and/or remedy disease. There would be a greater set of options for treating illness, and patients would be able to avoid exacerbating existing diseases.

\section{Language and culture-a path to new understanding}

In order for traditional Asian medicine to make a significant contribution to public health in the West, however, there are two conditions that need to be satisfied. First, we must have a language with which to explain our concepts, and we must have a culture that is opened to them. Those practising in fields of natural medicine must be able to express their ideas in a coherent manner to non-experts. Currently, when natural medicine practitioners speak about their disciplines, they are too often limited by their own jargon or to a misapplication of biomedical or scientific terminology. Therefore, much greater work needs to be done to create language that allows for a clear and constructive dialogue with lay people and between practitioners of different medical systems and traditions.

For example, the generalised use of the term 'energy' in natural medicine can often be confusing. If we mean to artificially limit the definition of the complex principles of function of the body and mind that are described in the theories of Asian medical systems and traditions to that which is defined as energy in Western science, then the phenomena must be measurable by specific means. However, research that attempts to measure and narrowly define phenomena such as $q i$ as energy is likely to obscure an understanding of its broader and more complex definition and thereby reinforce misunderstanding and skepticism. ${ }^{9}$ We need to ask ourselves if 'energy' is, in fact, what we are speaking about, or are we attempting to describe mind/body principles that are more complex in nature and might

${ }^{9}$ Harmanci 2006. 
only include energetics? If we want to see an improvement in the quality of scientific research being done on natural medicine, we must begin to ponder such key questions. ${ }^{10}$

The terminology of western science and biomedicine is specifically and narrowly defined and should be respected. If we allow our discussion of natural medicine to be constrained by the hegemony of that language, we will limit rather than expand the possibility for cross-disciplinary and cross-cultural dialogue. Therefore, instead of looking, for example, to Evidence Based Medicine and randomised controlled trials to establish our legitimacy, we need to insist on new research protocols that are appropriate for the study of the holism that defines traditional Asian medicine."

Practitioners of traditional medicine should become more sophisticated in the language of biomedicine and research, rather than often using it inaccurately as a way to be accepted in the biomedical industry, academic research environments and medical insurance systems. Lay people, biomedical physicians and researchers need to better appreciate the integrity of traditional Asian medical systems, rather than incorrectly seeing them as a collection of therapies that can be cut and pasted into the biomedical paradigm. Humanities and social science scholars who study traditional Asian medicine also need to take a much more active role in helping our colleagues in biomedicine and science to develop a sophisticated understanding about these medical traditions, especially when the latter group seeks to research traditional medical approaches.

Practitioners and scholars should work cooperatively to create an environment where traditional Asian medical ideas can be accurately and usefully integrated into Western sensibilities. There is a traditional Tibetan view about the prerequisites for successfully integrating new ideas into a given environment that can inform that process. A Tibetan proverb states that a student is like a vessel, and that knowledge is a kind of pure substance that is to be placed inside of it. If the vessel is upside down, the substance will not be able to be placed within. That is, if people are close-minded due to ignorance, they will be unable to successfully accept new ideas. If the vessel is broken, its loss of integrity will render it unable to serve the purpose

10 Tokar 2006.

11 Djulbegovic 2000, pp. 99-109; Bensing 2000, pp. 17-25; Timmermans 1997, pp. 273-305. 
at hand. Lastly, if the vessel is polluted the pure substance will become corrupted and lose its true nature.

We need to ask ourselves what kind of 'vessel' our western cultures have become regarding the acceptance of new ideas concerning health and medicine. To help us integrate a new perspective of health into global culture, we require a pro-pluralist path, such as existed in the populist alternative medicine movement in the US from the 1960s through the 1980s. That progressive path was effectively coopted, beginning in the 1990s on the heels of Dr David Eisenberg's famous article, published in the New England Journal of Medicine, documenting the great extent to which people were paying out of pocket for 'unconventional' medical care. ${ }^{12}$

The article's impact was widespread in the US, and the government, the biomedical and pharmaceutical industries and universitybased researchers were soon vying for a share of the money that the Eisenberg study claimed to be in the hands of those practising alternative medicine in the US. A new nutriceutical industry also grew in the US, supplanting the former, more environmentally and ethically responsible, herbal products industry. ${ }^{13}$ The American Complementary and Alternative Medicine (CAM) industry was born in this new environment, and has been characterised by its prioritisation of the integration of natural medicine into the biomedical and health insurance industries. CAM's 'integrative medicine' product exemplifies this goal and is at odds with the values of medical pluralism.

This new industrial formulation has resulted in an explosion of the commoditisation of natural medicine that encourages biopiracy worldwide and poses a threat to fragile indigenous medical systems. ${ }^{14}$ Internationally, CAM activists have tried to influence biomedical doctors in Asia, Africa, the Middle East and South America to adopt their integrative model. By attempting to so influence those regions, where systems and traditions of natural medicine originate, they have been expanding the hegemony of the medical industrial approach. ${ }^{15}$

In America, the CAM industry has expanded the availability of certain natural therapies, and some hospitals here now give their

\footnotetext{
12 Eisenberg 1993, pp. 246-52.

13 Burcher 2002, p. 23.

14 Adams 2002, pp. 666, 675-6; Janes 1995, pp. 6-39; Shiva 2001, pp. 283-9; Pordie 2002, pp. 68-74.

${ }_{15}$ Dumoff 2003, pp. 45-8; Complementary and Alternative Medicine at the NIH 2002, pp. 1-2.
} 
patients access to treatments including certain massage, meditation and Chinese acupuncture techniques. ${ }^{16}$ When these approaches aid patients, they are clearly worthwhile. However, it should also be acknowledged that such institutionalised procedures usually do not represent the full value of the natural medical systems and traditions from which they have been appropriated.

\section{Conclusion}

If we desire an expansion of healthcare availability, a healthier population, and a reduction in medical costs, a more appropriate path is required. Economics has a role, but it should not be the central driver for progress in medicine and science. ${ }^{17}$ An inappropriate emphasis on econometrics in medicine is, after all, what has created healthcare crises in America and other regions internationally.

We can gain inspiration from the history of Tibetan medicine, an integrative system that was created by the intentional expansion of medical pluralism. Priority should be placed upon educating our fellow citizens about the highly detailed, traditional understanding of health, and the causes of illness, that exist in systems of traditional Asian medicine, such as Tibetan medicine.

To regain the best path for natural medicine, practitioners need to emphasise broad-based collegiality within and between our disciplines. Such steps would allow us to forge progressive alliances with biomedicine without having to compromise the integrity of our own unique medical systems. Promoting the values of medical pluralism will help protect ancient medical systems and traditions like Tibetan medicine from the negative effects of integration, co-optation and biopiracy. Furthermore, it will allow us to fulfill our potential to contribute to changing our focus away from the commerce of disease and toward the advancement of health awareness. In doing so, we can rejuvenate in medicine that which His Holiness the Dalai Lama reminds us is required: a 'good heart ... that shows a genuine sense of care, compassion and concern' for all. ${ }^{18}$

${ }^{16}$ Cowley 2002, pp. 46-53; Garner-Wizard 2003.

17 Angell 2000, pp. 1516-18; Shah 2002, pp. 22-4.

18 Dalai Lama 1999, pp. 67-9. 


\section{References}

Adams, V. 2002, 'Randomized Controlled Crime: Postcolonial sciences in alternative medicine research', Social Studies of Science, 32: 666, 675-6.

Allen, M. 2006, 'The World Health Organization (WHO) and the United States', Acupuncture Today, 7: <http://www.acupuncturetoday.com/archives2006/sep/ 09allen.html

Angell, M. 2000, 'Is Academic Medicine For Sale?', The New England Journal of Medicine, 342: 1516-18.

Bensing J. 2000, 'Bridging the Gap. The separate worlds of evidence-based medicine and patient-centered medicine', Patient Education and Counseling, 39: 17-25.

Burcher S. and M-W. Ho 2002, 'Global Strategy for Traditional Medicine', Science In Society, 16: 23.

Clifford, T. 1990, Tibetan Buddhist Medicine and Psychiatry: The diamond healing, Maine: Samuel Weiser, Inc.

Cowley, G. 2002, 'Now, Integrative Care', Newesweek, 140.23: 46-53.

Dalai Lama, H. H. 1999, 'The Relevance of Tibetan Medicine Today', Alternative Therapies In Health And Medicine, 5.3: 67-9.

Dhonden, Y. 2000, Healing from the Source, Ithaca, New York: Snow Lion Publications.

Djulbegovic B., L. Morris and G. H. Lyman 2000, 'Evidentiary Challenges to Evidence-Based Medicine', Journal of Evaluation in Clinical Practice, 6.2: 99-109.

Donden, Y. 1986, Health Through Balance, Ithaca, New York: Snow Lion Publications.

Dumoff, A. 2003, 'ACM: An international perspective', Alternative/Complementary Therapies, 9.1: 45-8.

Eisenberg D. M., R. C. Kessler, C. Foster, F. E. Norlock, D. R. Calkins, and T. L. Delbanco 1993, 'Unconventional Medicine in the United States: Prevalence, costs and patterns of use', Nere England Journal of Medicine, 328.4: 246-52.

Garner-Wizard, M. 2003, 'Newsweek Runs Cover Story on Alternative Medicine', Herbclip, 31 July, HC 020531-237.

Glanze, W. D., K. N. Anderson and L. E. Anderson 1992, The Mosby Medical Encyclopedia. Revised ed., New York: Penguin.

Harmanci, R. 2006, 'Healthy Doubts: Wallace Sampson alternative medicine doesn't exist and acupuncture is useless, he says', San Francisco Chronicle, 31 August: <http://sfgate.com/cgi-bin/article.cgi?file=/c/a/2006/08/31/NSGDOIM5RJ1.DTL

Janes, C. R. 1995, 'The Transformations of Tibetan Medicine', Medical Anthropology Quarterly, 9.1: 6-39.

Miller, D. M. 2004, 'Understanding Codex Alimentarius and Its Impact On Health Freedom': <http://www.friendsoffreedominternational.org/Articles/Codex/Codex 8_12_04.pdf

OIHR Shares Expertise in Asia, Complementary and Alternative Medicine at the NIH, National Center for Complementary and Alternative Medicine 2002, 9.1: $1-2$.

Parfionovitch, Y., G. Dorje, and F. Meyer 1992, Tibetan Medical Paintings: Illustrations to the Blue Beryl Treatise of Sangye Gyamtso, New York: Harry N. Abrams, Inc.

Pordie L. and Brisard 2002, 'Himalayan Medical Heritage Facing its own Success: Focus on intellectual property', Ethnopharmacologia, 29: 68-74.

rGyud zhi, 1994, Dharamsala, India: Tibetan Medical and Astrological Institute.

Shah, S. 2002, 'Rx needed for Medical Journals: Drug companies influence research; they also affect what gets published', The Nation, 274.3: 22-4.

Shiva, V. 2001, 'Biopiracy: The theft of knowledge and resources', in B. Tokar (ed.), Redesigning Life?, London: Zed Books, 283-9.

Timmermans, S. and M. Berg 1997, 'Standardization in Action: Achieving local universality through medical protocols', Social Studies of Science, 27: 273-305. 
Tokar, E. 1998a, Ancient Wisdom-Modern healing, Ayuroïnana, 5.2: 37-42.

— 1998b, 'A Tibetan Medical Perspective on Irritable Bowel Syndrome: Building a means of discourse for integrative medicine', Alternative and Complementary Therapies, 4.5: 343-9.

2005, 'Fast Food and Obesity in America', Letter to the Editor, The New York Times, 7 July: 1504(53,268): A22.

2006, 'Transformation and Balance: The principles of Tibetan medicine in the context of American healthcare', Unified Energetics, 1.2, 47-51.

Tokar, E. and A. Vora 1998, 'Between Heaven and Earth: An introduction to various philosophies and approaches to medical care', American Medical Students Association's National Project on Alternative and Complementary Medicine, 1.1: 1-2.

- 1999, 'Seeing to the Distant Mountain: Diagnosis in Tibetan medicine', Alternative Therapies In Health And Medicine, 5.2: 50-8.

Trogawa, S. L. and S. Dorje 1984-9, 'History of Tibetan Medicine', Unpublished Lectures, New York: Boulder, CO.

WHO, WHO Strategy for Traditional Medicine 2002-2005, WHO/EDM/TRM, 2002.1.

Yonten P. 1989, 'The History of the Tibetan Medical System', Tibetan Medicine, 12: $32-51$. 